\title{
How genomic and developmental dynamics affect evolutionary processes
}

\author{
Gabriel Dover
}

\begin{abstract}
Summary
Evolutionary genetics is concerned with natural selection and neutral drift, to the virtual exclusion of almost everything else. In its current focus on DNA variation, it reduces phenotypes to symbols. Varying phenotypes, however, are the units of evolution, and, if we want a comprehensive theory of evolution, we need to consider both the internal and external evolutionary forces that shape the development of phenotypes. Genetic systems are redundant, modular and subject to a variety of genomic mechanisms of "turnover" (transposition, gene conversion, unequal crossingover, slippage and so on). As such the construction and spread of novel combinations of modules by turnover, in particular within gene promoters, contributes significantly to the evolution of phenotypes. Furthermore, redundancy, turnover and modularity lead to ever more complex networks of genetic interactions and ever more functions for a given module. The significant interaction between genomic turnover and natural selection leads to a molecular coevolution between interacting modules and hence facilitates the establishment of biological novelties. BioEssays 22: 1153-1159, 2000. (C) 2000 John Wiley \& Sons, Inc.
\end{abstract}

\section{From genotype to phenotype}

In his now classic book on The Genetics of the Evolutionary Process $^{(1)}$ Richard Lewontin dismissed the practice of evolutionary genetics in the following amusing terms:

\begin{abstract}
"For many years population genetics was an immensely rich and powerful theory with virtually no suitable facts on which to operate. It was like a complex and exquisite machine, designed to process a raw material that no one had succeeded in mining.......... For the most part the machine was left to the engineers, forever tinkering, forever making improvements, in anticipation of the day when it would be called upon to carry out full production.

Quite suddenly the situation has changed. The mother-lode has been tapped and facts in profusion have been poured into the hoppers of this theory machine. And from the other end has issued-nothing........ The machine cannot transform into a finished product the great volume of raw material that has been provided....... The entire relationship between the theory and the facts needs to be reconsidered."
\end{abstract}

Department of Genetics, University of Leicester, University Road, Leicester, LE1 7RH, UK.

Funding agency: The Wellcome Trust.
This description encapsulates Lewontin's chief, and as yet unrequited worry, that if we are to understand the role of genes in evolution then "context and interaction are of the essence". What he meant by this is that unless and until we uncover the "rules of transformation" that connect "genotype space" with "phenotype space" then we cannot seriously entertain, or be satisfied with, a gene-based theory of evolution. How an individual phenotype emerges and reproduces from a given unique set of genes inherited from its sexual parents is the central question of evolutionary theory: all the rest is subsidiary. The reproductive prowess of an individual, relative to its peers, is a unique quality dependent on the morphological, metabolic and behavioural aspects of its phenotype.

Ever since the first methodological breakthroughs into the measurement of genetic diversity by electrophoresis, either of proteins or DNA, followed by methods of direct readout of DNA sequences, evolutionary genetics has all but abandoned the nature of the beast that it is attempting to understand. Evolutionary biology is reduced to the anxiety of whether the patterns of genetic variation in a population (polymorphism) or between species (diversity) is due to adaptational selection or neutral drift, or some combination of the two. The complex, functional relationships between genetic variants and phenotypes, or between a given genetic variant and the rest of the genotype, are often more of an embarrassing digression than an important, relevant consideration. Furthermore, key discoveries over the past 30 years showing that there exist several mechanisms of genomic turnover that can aid in the evolutionary spread of variation are not part of the mathematical edifice by which evolutionary genetics is considered to explain the real world. In this article, I review these mechanisms and discuss how they contribute to the generation of phenotypes, upon which evolutionary processes act.

\section{So, what have we learned?}

It has been said famously that "nothing in biology makes sense except in the light of evolution" (Th. Dobzhansky). To which truism we can add that nothing in evolution makes sense except in the light of the genome and development. Hence, it is necessary to consider what are the discoveries of the past 2030 years in genomic and developmental biology that could change the way that we think about selection, adaptation, speciation, sex and neutrality.

I believe that the three key features are non-Mendelian genetic turnover, redundancy and modularity. These are a 
mouthful to reiterate so I will use the acronym TRAM (Turnover; Redundancy And Modularity). So far as I'm aware, all genetic and developmental systems examined to-date have TRAM characteristics.

\section{Turnover}

DNA is a far more unstable molecule, on an evolutionary scale, than is conventionally thought. It is subjected to a variety of non-Mendelian mechanisms of turnover, over and above the mechanisms responsible for base-base substitutions. These genomic mechanisms are gene conversion, unequal crossingover, slippage, transposition, retrotransposition and so on. They are ubiquitous in all eukaryotic, archaebacterial and prokaryotic genomes and occur at rates that are often several orders of magnitude faster than the calculated rates of point mutations (for reviews see Refs 2,3). They are non-Mendelian in the sense that they can demote or promote the representation of a variant sequence within a genome; they are intrinsically distinct from selection and drift, which operate on populations. Hence, they can lead, in the fullness of time, to the spread of a variant sequence through a sexual population, a process termed "molecular drive". Imagine a gene conversion event between two distinct alleles of a gene such that the two alleles acquire the exact same sequence $(A a \rightarrow A A$; or $\mathrm{Aa} \rightarrow \mathrm{aa}$ ). This represents an homogenisation i.e., an increase in one allele ( $\mathrm{A}$ or $\mathrm{a}$ ) at the expense of the other. Now comes sex, after which the two homologous chromosomes enter two new individuals at the next generation, in each of which homogenisation by gene conversion may occur again. If gene conversion is biased in favour of one allele, then it will spread more rapidly through a population than if there is no bias in either direction.

It is wrong to assume that turnover mechanisms are simply more exotic ways of generating variation for selection or drift to play with. If we remain with gene conversion as an example, we need to know that the length of conversion can range from tens of kilobases (encompassing several genes) or involve only a few base pairs. In the latter instance, many small conversion domains generate mosaic genes. Hence, at the level of the longer unit of the gene, frequent microconversion generates variation; however, at the level of the smaller conversion domain, it leads to homogenisation. Hence, the representation of any mutation lying within the domain of conversion can increase or decrease in a sexual population, independently, initially at least, of selection or drift.

Classical evolutionary genetics is predicated on the premise that the Mendelian rules of inheritance cannot in themselves spread a mutation through a population with the passing of the generations. This can only be done either by selection or drift. The ubiquitous mechanisms of turnover offer a third mode of spread. Molecular drive is an umbrella term covering a number of turnover mechanisms that generate a bewildering variety of sequence patterns, both within and between populations. Whilst some consideration has been given by evolutionary geneticists to the phenomenon of "meiotic drive" (often the biased segregation of a chromosome over its homologue; see Presgraves and Orr, this volume), meiotic drive is just the tip of the iceberg of molecular drive: the results of collective mechanisms of non-Mendelian turnover occurring throughout a genome. I know of no sequences, when analysed in full, that are refractory to one or other mechanism of turnover.

\section{Redundancy}

The vast majority of DNA sequences are redundant at one level or another. "Redundancy" in genetics, however, has two meanings: existing in multiple copies or being superfluous to function. The two meanings are not synonymous in that a multiple-copy DNA sequence can be a functional multigene family. Different forms of DNA redundancy are generated by a variety of turnover mechanisms. The unit of repeat can be as small as one base pair (as generated by the stuttering process of slippage) or involve a whole set of chromosomes (as generated by cellular misdivision during mitosis and sometimes meiosis). Importantly, redundancy exists in the majority of gene promoters in which a variety of DNA sites bound by regulatory proteins exist in multiple copies. I shall focus later on the significance of promoter restructuring by turnover and the role of redundancy.

\section{Beyond Ohno}

The late Susumo Ohno wrote his classic book Evolution by Gene Duplication in 1970. ${ }^{(4)}$ His main premise is that, after a duplication event (by one or other mechanism of turnover), selection can hold one gene steady for an existing function whilst the duplicate is free to mutate further and be promoted by selection should it develop an advantageous new function. Evolutionary genetics has made some attempts to incorporate this phenomenon into evolution theory, mainly by Tomoko Ohta. ${ }^{(5)}$ The phenomenon in itself dominates the thinking of developmental geneticists when telling evolutionary stories. In many cases of genetic duplications with their sequence and functional diversity, Ohno's idea of keeping one gene locked whilst the other can mutate seems to be the most plausible explanation. For example, it is often suggested as a mechanism for interpreting the evolutionary history of clusters of similar but not identical globin genes or Hox genes amongst species. Close inspection, however, for instance in globins, reveals that gene conversion can occur between two duplicates (or parts of duplicates) leading to homogenisation (reviewed in Ref. 3). Hence, the unit of consideration needs to be the "cross-talking" gene pair or parts of gene pairs. The same is true of many other gene families such as the immunoglobulin genes and the genes at the MHC locus, repeatedly undergoing new rounds of homogenisation on an evolutionary timescale. We also need to bear in mind that the

\section{BioEssays 22.12}


unit of selection is the individual phenotype not the gene: hence, I have problems in understanding how selection "knows" which gene to conserve and which gene to allow to diverge in the Ohno concept. An easy way out is to ignore phenotype and to assume that each gene is "looking after itself" with regards to its specific evolutionary trajectory. But that's cheating and ultimately unsatisfactory. I return to this problem below.

If we have difficulties in monitoring one pair of genes or parts of genes, then consideration of multiple genes undergoing a variety of rearrangements and homogenisation through mechanisms of turnover, operating on a variety of unit lengths of sequence, and often one on top of another, becomes a major problem. Nevertheless, the challenge is there for the taking. This challenge is particularly daunting when we consider the phenomenon of modularity.

\section{Modularity}

The importance of modularity in biology is now firmly established (for review see Ref. 6). A module is an independent unit or process or function that may interact in a variety of combinatorial interactions with a variety of other units or processes or functions. Modules can be as small as a few base pairs (e.g. binding sites in gene promoters) or a small number of amino-acids (e.g. the homeodomain), and as large as a complete cellular structure (e.g., the ribosome) or a cellular and developmental process (e.g., signal transduction and DNA replication). At the protein level, it has been calculated that there may be as few as 1500-2000 modules that give rise to tens of thousands of mosaic proteins. ${ }^{(7,8)}$

Modularity at the genetic level has important evolutionary consequences. To illustrate this, I turn to the promoters of genes which are TRAM systems par excellence, and which play a key role in the evolution of biological novelties and new species.

In Fig. 1, the promoter regions of three genes in Drosophila melanogaster involved with early development of the larva, in addition to other functions later in life, are depicted. There are three significant features, all of which can be found in the promoters of most genes in most organisms (for review see Ref. 9). (1) A promoter can consist of a number of unrelated modular binding sites recognisable by a number of unrelated trans-acting regulatory proteins. (2) A given binding site can be shared by the promoters of unrelated genes. (3) The number and orientation of a given binding site can differ between promoters.

Figure 2 illustrates an example of a promoter of a given gene (in this case, the "gap" segmentation gene hunchback) as it exists in four different species of higher dipteran flies. This comparison shows differences in the number, orientation, and spacing of the binding sites recognised by the homeodomain of the bicoid regulatory protein. The consensus sequence of the sites also differs between the species.

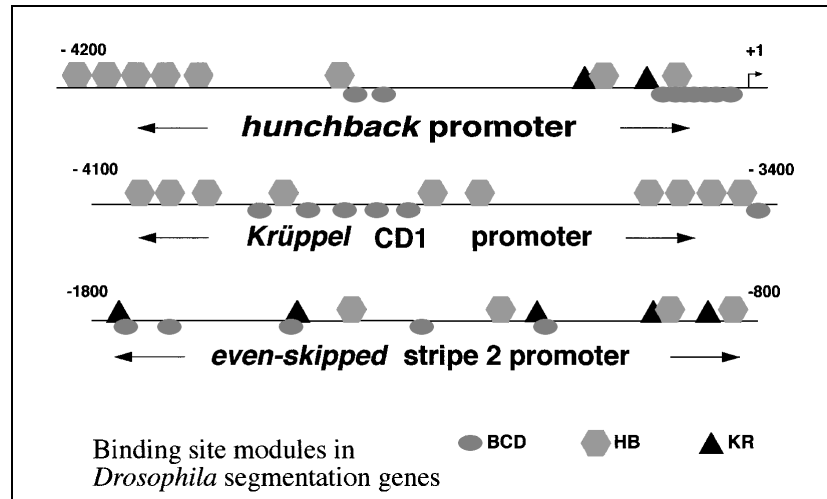

Figure 1. The promoters of three unrelated genes hunchback $(h b), K r u p p e l(K r)$ and even-skipped (eve) are depicted upstream from their respective genes. Each promoter contains a number of target sites to which regulatory proteins bind, repressing or activating the transcription of the downstream gene. The binding sites are symbolised in three ways, as shown, for the three regulatory proteins, BICOID (BCD), HUNCHBACK (HB) and KRUPPEL (KR). Note (i) binding sites are in varying numbers of copies due to genomic turnover; (ii) binding sites are shared by unrelated promoters, hence must be mobile; (iii) a gene's level of transcription is a reflection of combinatorial interactions amongst the different numbers and types of modular binding sites.

The promoters illustrated in Figs. 1 and 2 are simple relative to the complex, compound promoters of genes such as the Hox gene Ubx in D. melanogaster (reviewed in Ref. 10), and many of the genes from the diverse animal and plant species (reviewed in Ref. 9). Nevertheless, these more simple promoters have TRAM characteristics. (1) They are subject to mechanisms of genomic turnover (transposition; slippage; gene conversion; unequal crossingover etc). In the absence of such mechanisms it is difficult, if not impossible, to explain the extensive sharing of binding sites between unrelated genes, coupled to intergenic and interspecific differences in the number, orientation, spacing, and consensus sequences of the sites. The restructuring of promoters on an evolutionary timescale and extensive cross-gene sharing of modular binding sites is unlikely to have occurred by coincidence of occurrence of single point mutations and/or indels. For evidence on the involvement of slippage and other mechanisms in the evolution of the hunchback promoter in diverse species see Refs 11,12 . (2) The binding sites are redundant both in number and in function. For example, not all the existing binding sites in the hunchback promoter of $D$. melanogaster share parity in the extent to which they are required for hunchback regulation. The same is true of other examined promoters, which can contain a minimum central "core" of essential sites from the total number available. (3) The binding sites are modular. By this I mean that they, either singly or as small clusters, are relatively independent units of function 


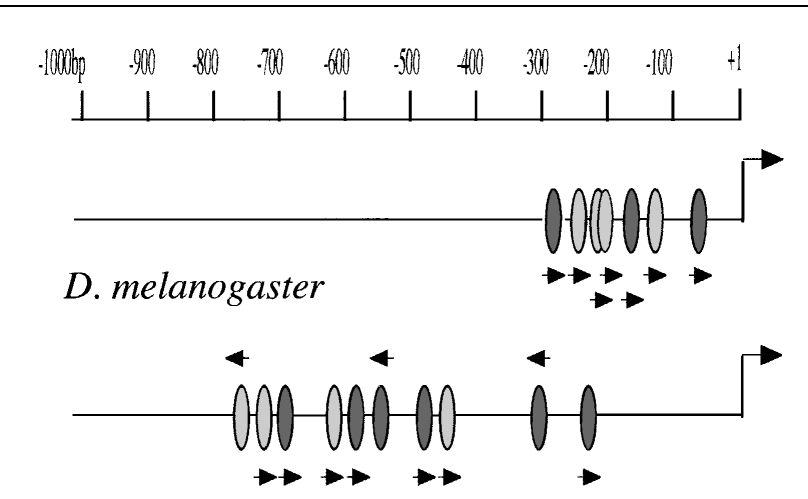

M. domestica

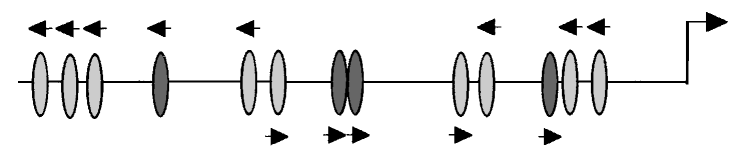

L. sericata

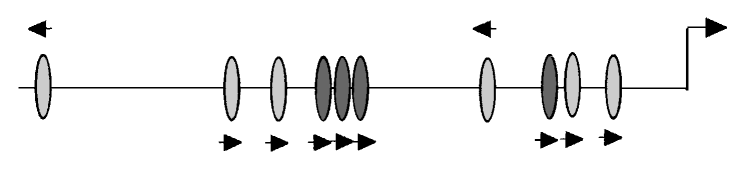

C. vicina

Figure 2. A comparison of the distribution, number, orientation and strength of binding of bicoid-binding sites in the hunchback promoter of four species of higher dipteran flies from the genera Drosophila, Musca, Lucillia and Calliphora. Filled oblongs are strong binding sites and hatched oblongs are weak binding sites. The binding sites in $L$. sericata and $C$. vicina have not yet been fully assayed (work in progress). Only the known functionally active sites in $D$. melanogaster and $M$. domestica are shown (see Refs 11,12).

often with short-range effects. Experiments by M. Levine and co-workers $^{(13)}$ and E. Davidson and co-workers ${ }^{(14)}$ have shown that new compound promoters can be experimentally created by shifting a section of the promoter of one gene to the promoter of another gene. Such artificially restructured promoters often lead to the novel, additional regulation of the recipient gene at times and in places expected of the incoming module of information from the donor gene's promoter. The transposition of modular information from one gene to another, as a new gene comes under the influence of a previously inaccessible regulatory protein, does not cause the complete breakdown of the pre-existing promoter. If this is the outcome in experimental time, then it is not inconceivable that functionally novel, restructured promoters arose by similar mechanisms of turnover causing the movement of modules and leading to the co-option of genes into new regulatory circuits (for extension of this argument see Ref. 2).
Turnover, redundancy and modularity are all intimately linked in their effects and I do not wish to create arbitrary distinctions. For example, new combinatorial interactions between trans- and cis-acting elements in gene regulation can become successfully established in populations of individuals because (i) turnover can lead to molecular drive, that is, the spread of a novel structure by, for example, transposition or gene conversion in a sexual population ${ }^{(3)}$, (ii) redundancy can lead to genetic and functional buffering as a number of modules continue to function whilst others mutate or move around, and (iii) modularity can lead to the creation of more complex promoters without the destruction of preexisting functions. The evolution of TRAM promoters can be envisaged as analogous to a more and more complex Lego construction, using novel combinations of relatively few basic building blocks-in this case-regulatory proteinbinding sites.

Although I have focussed on promoters, the same features of genetic construction and potential evolutionary progression apply to many other TRAM systems in the cell and organism. Such systems can lead to the creation of extensive networks of interactions as genes gain new functions. In reality there can be one gene influencing many functions, and one function with many genetic inputs.

The following few examples illustrate the extensive sharing of modules in unrelated processes. This sharing is becoming regarded as a basic common component of biological organisation (for further discussion see Ref. 15). For example, the bicoid gene in $D$. melanogaster is involved with the regulation of at least a dozen different promoters, each containing multiple bicoid-binding sites acquired through the independent movements of bicoid-binding modules around the genome. Members of the Wnt gene family encoding secreted glycoproteins not only participate in many signalling events during development, but also are involved with a number of extracellular and cell-surface proteins involved in cell-fate assignment, cell adhesion and metabolism. Similarly, many of the intracellular components of Wnt signalling are involved with other cellular functions. The high degree of pleiotropy of Wnt proteins, as they participate in many developmental processes, raises the question, posed by Martinez Arias and colleagues, whether Wnt signalling is better described as a network of interactions rather than a simple pathway of cause and effect. ${ }^{(16)}$ Finally, the long-time conservation of the regulatory circuit controlling eye development, involving the Pax6 gene, is a well-known story. ${ }^{(17)}$ Of more recent interest is the finding that the same combination of transcriptional regulators is redeployed elsewhere during vertebrate development of somite and skeletal muscle derivatives. ${ }^{(18)}$ Indeed, the phenomenon of modularity begins down at the level of the Pax genes, which comprise a small redundant family of genes that differ one from another, both within and between species, by varying combinations of three

\section{BioEssays 22.12}


modules: a homeodomain; the "paired" module and the "octapeptide" module.

\section{Robustness and flexibility in evolution}

A simple case of tolerance is the duplicate pair of genes in which regions of shared sequence, either as remnants of the original duplication or through a recent conversion event, are able to compensate functionally one for the other in the face of new mutations. Regardless of whether compensation is "full" or "partial", there is a need to consider the genetic and functional relationships between the genes, as a pair, in models of evolution, (for reviews see Refs 19,20).

There is increasing evidence that complex interactive genetic systems are "robust"-defined as the ability of a system to continue functioning despite substantial changes to its components. In more complex systems, surprising robustness has been uncovered in the genetic switch of phage $-\lambda$ (lambda) responsible for the qualitative change of state from lysogeny to lysis. This switch involves a complex promoter lying between two key autoregulatory genes having different affinities for different modular binding sites in the promoter. The normal pattern of binding has been experimentally altered such that the binding sites have identical sequences. ${ }^{(21)}$ Somewhat counter-intuitively, the variant phages had the same qualitative in vivo patterns of gene expression as the wild type. Although the precise mechanism by which robustness is achieved in this instance is unknown, nevertheless its existence in such a complex regulatory circuit underlines the point that evolution might be easier than we think. We do not have to consider the bringing together over evolutionary time of all the "right" components; rather there are a number of alternative combinations that can give rise to the same phenotypic output.

Much of the discussion of the evolutionary establishment of robustness is discussed within the context of selection acting alone. ${ }^{(20,21)}$ This could be a correct point of view, but it is probably too narrowly based in that TRAM genetic systems have additional exploitable features, as exhibited in the phenomenon of molecular coevolution, for the successful establishment of the twin evolutionary concerns of "robustness" and "flexibility".

\section{Molecular coevolution}

Ontogeny cannot be changed at will. There is a requirement to produce individuals that can reproduce their own kind-not precisely their own type, for sexual shuffling ensures that identity by descent cannot occur-nevertheless, there needs to be a continuity of successful genetic processes through the generations.

It is generally assumed that natural selection would eventually dispose of any biological difficulties in the production of successfully reproducing phenotypes. On this basis, reconstructed promoters with TRAM features and dynamics can be expected to be no exception. We could argue, within the strict paradigm of evolution by natural selection acting alone, that no matter how internally buffered an organism might be (given redundancy, turnover and modularity), the future representation of a novel promoter in a population depends on its effects on the relative reproductive success of the individual phenotype. If it fails to work-let's demote it. If it works better than ever-let's promote it. If it's neither one nor the other then let it run to extinction or to fixation according to the stochastic processes that govern neutral drift.

There is, however, a third window of opportunity for selection that does not involve outright promotion or demotion of the reconstructed promoter. This is for selection to promote compensatory changes, either in the genes producing the trans-acting regulatory proteins or in flanking cis-acting genetic elements also involved with gene regulation. In other words, a good deal of the responsibility imposed on selection is to promote co-evolutionary changes between two genetic partners involved in a given functional interaction. When mechanisms of turnover create and begin to spread novel promoter constructs through a small sexual population of related individuals, selection has the opportunity to screen the population for variant alleles of, say, trans-acting regulatory genes which are better able to interact with the new set of reconstructed promoters. Such a molecular coevolution between trans and cis partners involved with gene regulation was first observed in the rDNA system. ${ }^{(22)}$

Molecular coevolution in this TRAM system has led, for example, to the failure of the Pol I transcription machinery of $D$. melanogaster to recognise the rDNA promoters of $D$. hydei. The same is true in many other groups of animals and plants. Similarly, a coevolution of homeodomain sequences and their binding sites has been reported for a variety of developmental genes $^{(23)}$ (and see Ref. 24 for review) and between adjacent interactive cis-elements in cases such as the per clock gene and the Adh gene in Drosophila. ${ }^{(25,26)}$ Figure 3 illustrates the phenomenon in its simplest form.

Interestingly, one of the first documented examples of molecular coevolution among promoters of developmental genes involved the two genes fushi-tarazu (ftz) and bicoid. ${ }^{(23)}$ Both have the sixty amino acid homeodomain module, but at amino acid position number 50 , the $\mathrm{ftz}$ protein has the amino acid glutamine and the bicoid protein has lysine. There is a corresponding difference in their respective binding sites in the promoters of several other genes. The $f t z$-binding site has a pair of CC nucleotides at the start (CCATTA), whereas the bicoid-binding side starts with GG in the same positions. When the glutamine of $\mathrm{ftz}$ is replaced artificially by the lysine of bicoid, the $f t z$ protein seeks out the GG-containing binding sites of bicoid. Clearly the glutamine-CC and the lysine-GG couplings are both perfectly functional, indicating molecular coevolution. Given the multiple binding sites shared by unrelated promoters, we can expect molecular drive to have 


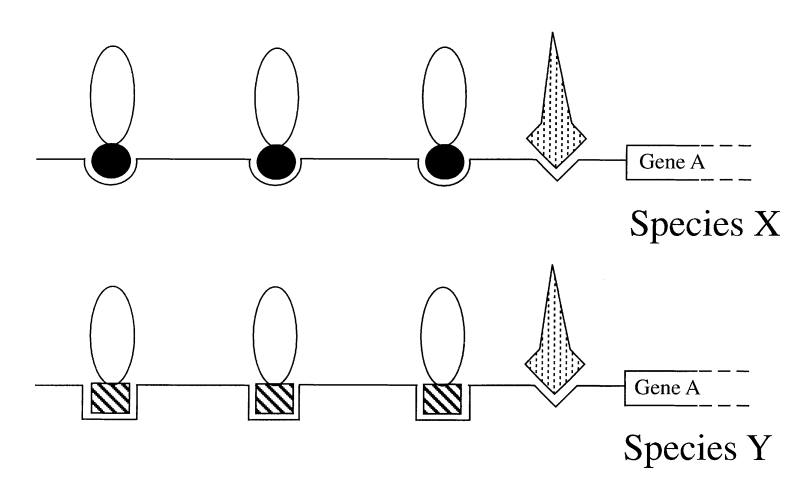

Figure 3. Molecular coevolution of regulatory protein and promoter binding sites. Two species, $X$ and $Y$, share the same gene, A. Each gene has a promoter consisting of three copies of a binding site to a regulatory protein. However, the molecular matching between the binding sites and the protein is different between the species. This is shown by slightly different shapes and shading. The regulatory protein of species $X$ binds with too much or too little efficiency, or not at all, to the binding sites of species $\mathrm{Y}$, and vice versa. Another regulatory protein and another unchanged binding site are shown. If species $X$ and $Y$ are very closely related, molecular coevolution between a protein and its binding sites might not yet have become established. spread new variant sites leaving selection to promote compensatory mutations in the homeodomains.

Non-Mendelian mechanisms of turnover ensure, through their capacity to influence the frequency of novel promoters in a population, that the evolutionary trajectory of a promoter is not governed solely by the forces that underpin selection. The non-Mendelian mechanisms can create novel genetic variants and, at the same time, aid in the spread of one novel variant or another. ${ }^{(3)}$ This internally driven process is operationally distinct from natural selection; hence, the involvement of selection in the acceptance or rejection of a novel promoter would require either some direct selective interference with genomic turnover and its homogenising consequences, or be engaged with the promotion of compensatory changes in other relevant genes. ${ }^{(22)}$ Given that, as far as we know, the ubiquitous non-Mendelian mechanisms never cease putting genomes through a continual state of flux on an evolutionary timescale, and given that molecular coevolution is often observed between interacting partners wherever this is looked for in interspecific or intertaxa comparisons, then the latter option seems to be the route favoured by selection.

Redundancy, modularity and turnover coupled to selective molecular coevolution means that subtle differences can accumulate between separated populations regarding the molecular nuts-and-bolts by which interactions take place; for example, in the way transcription factors recognise their binding sites. Hence, the phenomenon of molecular coevolu- tion ensures that the biological function of a given interaction is maintained, whilst subtly reconstructed promoters may accumulate leading ultimately to biological incompatibilities and the rise of new species. I have likened this process of internal coevolution to the unique trick, learned by evolving biological organisms, of how to change the design and construction of an aeroplane while it is still flying. ${ }^{(2)}$

\section{"Context and interaction are of the essence"; Lewontin}

The evolution of new species-specific ontogenies is a result of new combinatorial interactions between existing genetic modules, rather than selective increase of new genes for new jobs. Evolution is about teaching old modules new tricks (for further discussion see Ref. 15).

At the same time, we can see that any given part of phenotype has multiple genetic inputs. There is a many-onmany relationship between genotype and phenotype: not a one-to-one relationship between gene and part. Hence, the gene, in itself, is "ignorant" of its role in development: it will inevitably interact with other genes at a given time and place depending on the developmental history of the interactions up to the point in question, which in turn is dependent on the evolutionary history of events in a particular lineage of genomes. From this perspective, one can see how misleading it is to speak of "blueprints", "recipes", and "instructions" in biological systems. There is a time-dependent unfolding of massively complex genetic interactions involving a relatively small number of modular yet highly promiscuous units. The combination of the internal forces in the genome, as they involve TRAM genetic systems, and the external forces of the natural ecology, cannot be modelled by concepts that embrace the latter to the exclusion of the former. We need to understand the key parameters of modularity, redundancy and turnover, with their attendant effects of combinatorial promiscuity, genetic networks, tolerance, and molecular coevolution, in particular in regulatory genetic systems, before we can assume that we have a comprehensive theory of evolution. There have been some useful starts in this direction, at least with regards to turnover and redundancy (for references see Refs. 27,28$)$ and with regards to epistasis and pleiotropy, $(29,30)$ but there is a long way to go.

\section{References}

1. Lewontin RC. The Genetic Basis of Evolutionary Change. Columbia University Press 1974. p 337.

2. Dover GA. Dear Mr Darwin; Letters on the Evolution of Life and Human Nature. Weidenfeld and Nicholson and University of California Press. 2000. p 268.

3. Dover GA. Molecular drive: a cohesive mode of species evolution. Nature 1982;299:111-117

4. Ohno S. Evolution By Gene Duplication. New York: Springer-Verlag. 1970

5. Ohta T. Selected Papers on Theoretical Population Genetics and Molecular Evolution. Natl Instit Genetics, Mishima Japan. 1997. p 404

\section{BioEssays 22.12}


6. Hartwell LH, Hopfield JJ, Leibler S, Murray AW. From molecular to modular cell biology. Nature 1999;402:C47-C52.

7. Bork P. Mobile modules and motifs. Curr Biol 1992;2:413-421.

8. Chothia C. Protein families in the metazoan genome. In Akam M, Holland P, Ingham P, Wray G. ed; The Evolution of Developmental Mechanisms (Development Supplement 1994). Cambridge, UK: The Company of Biologists. 1994. p 27-33.

9. Arnone MI, Davidson EH. The hardwiring of development: Organisation and function of genomic regulatory systems. Development 1997;124 $1851-1864$

10. Akam M. Hox genes: From master genes to micromanagers. Curr Bio 1998;R676-R678.

11. Hancock JM, Shaw $P$, Bonneton F, Dover GA. High sequence turnover in promoters of the developmental gene hunchback in insects. Mol Biol Evol 1999;16:253-265.

12. Bonneton F, Shaw P, Fazakerley C, Shi M, Dover GA. Comparison of bicoid-dependent regulation of huncback between Musca domestica and Drosophila melanogaster. Mech Dev 1997;66:143156

13. Gray S, Levine M. Transcriptional repression in development. Curr Opin Cell Biol 1996;8:358-364

14. Kirchhamer CV, Bogarad LD, Davidson EH. Developmental expression of synthetic cis-regulatory systems composed of spatial control elements from two different genes. Proc Natl Acad Sci USA 1996;93:1384513849.

15. Duboule D, Wilkins AS. The evolution of "bricolage". Trends Genet 1998; 14:54-59

16. Martinez Arias A, Brown AMC, Brennan K. Wnt signalling: pathway or network? Curr Opin Dev Biol 1999;2:447-454.

17. Gehring WJ, Ikeo K. Pax 6: Mastering eye morphogenesis and eye evolution: Trends Genet 1999;371-377
18. Pelaic F, Buckingham M. From insect eye to vertebrate muscle: rede polyment of a regulatory network. Genes Dev 1999;13:3171-3178.

19. Wagner A. Robustness against mutations in genetic networks of yeast. Nature Genetics 2000;24:355-361.

20. Wilkins AS. Canalization: a molecular genetic perspective. Bioessays 1997; 19:257-262.

21. Little JW, Shapley DP, Wert DW. Robustness of a gene regulatory circuit. EMBO J 1999;18:4299-4307.

22. Dover GA, Flavell RB. Molecular coevolution: DNA divergence and the maintenance of function. Cell 1984;38:623-624.

23. Percival-Smith A, Muller A, Affolter M, Gehring WJ. The interaction with DNA of wild-type and mutant fushi-tarazu homeodomains. EMBO J 1990;9:3967-3974.

24. Dover GA. Observing development through evolutionary eyes: a practical approach to molecular coevolution. Bioessays (Special Issue: Evolution and Development) 1992;14:281-287.

25. Peixoto AA, Hennessy JM, Townson I, Hasan G, Rosbash M, Costa R, Kyriacou CP. Molecular coevolution within a Drosophila clock gene. Proc Natl Acad Sci USA 1998;95:4475-4480.

26. Ludwig $\mathrm{MZ}$, Bergman $\mathrm{C}$, Patel $\mathrm{NH}$, Kreitman M. Evidence for stabilizing selection in a eukaryotic enhancer element. Nature 2000;403:564-567.

27. Ohta T, Dover GA. The cohesive population genetics of molecular drive. Genetics 1984;108:501-521.

28. Ohta, Dover GA. Population genetics of multiple families that are dispersed into two or more chromosomes. Proc Natl Acad Sci USA 1993; 80:4079-4083.

29. Gibson G. Epistasis and pleiotropy as natural properties of transcriptional regulation. Theoret Pop Biol 1996;49:58-89.

30. Gibson G, Hogness DS. Effect of polymorphism in the Drosophila regulatory gene Ultrabithorax on homeotic stability. Science 1996;271: 200-203 